\title{
Gene expression profiles in esophageal adenocarcinoma predict survival after resection
}

\author{
Arjun Pennathur, MD, ${ }^{\mathrm{a}}$ Liqiang Xi, MD, ${ }^{\mathrm{b}}$ Virginia R. Litle, MD, ${ }^{\mathrm{c}}$ William E. Gooding, MS, ${ }^{\mathrm{d}}$ \\ Alyssa Krasinskas, MD, ${ }^{\mathrm{e}}$ Rodney J. Landreneau, MD, ${ }^{\mathrm{a}}$ Tony E. Godfrey, $\mathrm{PhD},{ }^{\mathrm{c}}$ and James D. Luketich, $\mathrm{MD}^{\mathrm{a}}$
}

Objective: The incidence of esophageal adenocarcinoma is rapidly increasing in the western population. Despite aggressive treatment, survival after esophagectomy is suboptimal. The main objective of the present study was to evaluate the gene expression profiles in esophageal adenocarcinoma and determine their association with survival after resection.

Methods: We conducted a prospective National Institutes of Health/National Cancer Institute funded study to evaluate the prognostic significance of gene expression in patients with esophageal adenocarcinoma undergoing esophagectomy. Gene expression in tumor tissue was analyzed using high-throughput oligonucleotide arrays. The association of gene expression and overall survival was analyzed using the tail-strength statistic and Cox regression analysis. Gene signatures were constructed with semisupervised methods using principal components. A cross-validated risk score was devised by conducting 10-fold cross-validation, 100 times.

Results: We evaluated the gene expression in 64 patients with esophageal adenocarcinoma who underwent esophagectomy. The median overall survival was 27 months ( $95 \%$ confidence interval 22 to not reached). After filtering, 10,214 probe sets were used for survival analysis. The tail-strength statistic for these probe sets (0.318) indicated a significant association with overall survival. Patients were classified into high- and low-risk groups, according to the gene signature. High-risk patients had a predicted median survival of 19 months, but the median was not reached for the low-risk group $(P<.05)$. On multivariate analysis, the gene signature was independently associated with survival (hazard ratio, $2.22 ; P=.04$ ).

Conclusions: Global gene expression levels were significantly associated with overall survival after esophagectomy. Furthermore, individual genes could be successfully combined into a strongly predictive, internally cross-validated gene signature. If validated further, these results could help direct additional clinical trials of neoadjuvant and adjuvant therapies for esophageal adenocarcinoma. ( $\mathrm{J}$ Thorac Cardiovasc Surg 2013;145:505-13)

The incidence of esophageal cancer has been dramatically increasing during the past 3 decades. ${ }^{1,2}$ In the United States and the western population, this profound increase has resulted from an increase in the incidence of adenocarcinoma of the esophagus. The incidence of esophageal adenocarcinoma (EAC) now exceeds that of squamous cell carcinoma in the United States and the west. This major epidemiologic shift is thought to be

From the Department of Cardiothoracic Surgery, ${ }^{a}$ University of Pittsburgh Medical Center, Pittsburgh, Pa; Laboratory of Pathology, ${ }^{\mathrm{b}}$ Center for Cancer Research, National Cancer Institute, National Institutes of Health, Bethesda, Md; Department of Surgery, ${ }^{c}$ University of Rochester Medical Center, Rochester, NY; Biostatistics Facility, ${ }^{\mathrm{d}}$ University of Pittsburgh Cancer Institute, Pittsburgh, Pa; and Department of Pathology, ${ }^{\mathrm{e}}$ University of Pittsburgh, Pittsburgh, Pa

This work was supported by National Cancer Institute grant 5R01CA090665.

Disclosures: Authors have nothing to disclose with regard to commercial support.

Presented in part at the 90th Annual Meeting of The American Association for Thoracic Surgery, Toronto, Ontario, Canada, May 1-5, 2010.

Drs Tony E. Godfrey and James D. Luketich contributed equally to the study.

Received for publication Dec 6, 2010; revisions received Sept 28, 2012; accepted for publication Oct 22, 2012.

Address for reprints: James D. Luketich, MD, Department of Cardiothoracic Surgery,

University of Pittsburgh Medical Center, 200 Lothrop St, C-800, Pittsburgh, PA

15213 (E-mail: luketichjd@upmc.edu).

$0022-5223 / \$ 36.00$

Copyright (c) 2013 by The American Association for Thoracic Surgery

http://dx.doi.org/10.1016/j.jtcvs.2012.10.031 related to gastroesophageal reflux disease, obesity, and Barrett's esophagus (BE), which are the dominant risk factors. ${ }^{2}$ In the United States, the estimated incidence of esophageal cancer was more than 16,000 cases in $2009 .^{1}$ The overall outcome after the diagnosis of esophageal cancer is suboptimal, with a 5-year survival rate of $15 \%$ to $25 \%$, although an improvement in survival, associated with early-stage disease, was seen in recent surgical series. ${ }^{3}$

Accurate staging before treatment is important and facilitates the selection of appropriate treatment strategies. Despite improvements, however, the current clinical staging modalities have not proved very accurate. ${ }^{4-6} \mathrm{~A}$ better understanding of the biologic behavior of the tumor will aid in the selection of appropriate therapies, with the potential to improve the outcomes of those with esophageal cancer. The analysis of the gene expression profiles associated with different outcomes could potentially be useful for the careful selection of therapies and could also aid in tailoring treatment to the individual patient. ${ }^{7,8}$

Although the expression of single genes or specific pathways have been studied in the pathophysiology of cancer, ${ }^{9}$ it is clear that the pathogenesis and progression of cancer is a complex process involving many genes and pathways. 


\section{Abbreviations and Acronyms \\ $\mathrm{BE}=$ Barrett's esophagus \\ $\mathrm{CI}=$ confidence interval \\ $\mathrm{EAC}=$ esophageal adenocarcinoma \\ $\mathrm{HR}=$ hazard ratio}

Gene expression profiling with microarrays is a powerful and promising modality for evaluating the expression of a large number of genes and evaluating changes in genomewide expression. ${ }^{8}$ The gene expression pattern of the primary tumor has been shown to predict the outcome for several malignancies, including lung cancer, head and neck cancer, and breast cancer. ${ }^{10-13}$ Several studies have explored gene expression in squamous cell cancer of the esophagus, and differential gene expression between esophageal cancer cell types has also been evaluated. However, currently, studies evaluating the prognostic implications of genome-wide changes in the gene expression for EAC are limited. ${ }^{14-19}$

Our main hypothesis was that the gene expression patterns observed in EAC would be associated with survival after surgical resection. We hypothesized that a set of genes or gene profile would be predictive of survival after surgical resection in patients with EAC. The main objective of the present study was, therefore, to evaluate the gene expression profiles of EAC, identify individual genes associated with outcome, and build a gene expression signature to identify high- and low-risk patients.

\section{METHODS \\ Patients}

We conducted a prospective National Institutes of Health, National Cancer Institute-funded study to evaluate the prognostic significance of gene expression profiles in EAC. The institutional review boards at the University of Pittsburgh and other participating institutions approved the study protocol. All patients enrolled in the present study provided informed consent. Tumor tissues were prospectively collected from eligible patients who had provided informed consent prior to undergoing esophagectomy for EAC of the esophagus at the University of Pittsburgh Medical Center from 2002 to 2006. The present study involved prospective patient consent, tissue and data collection, and correlative analysis with gene expression. More details on the tissue collection and processing and gene expression analysis are provided under individual subheadings in this section. Patient inclusion criteria included histologically proven adenocarcinoma, complete surgical resection with negative margins, and the absence of induction therapy. Patients were excluded if they were found to have distant metastatic disease on surgical exploration or if they died within 30 days of the operation date. Computed tomography, endoscopic ultrasonography, minimally invasive staging, and positron emission tomography were used for staging. We have previously shown that minimally invasive staging is more accurate than positron emission tomography for the detection of distant metastasis. ${ }^{4}$ Patients underwent disease staging with computed tomography $(64 / 64 ; 100 \%)$, endoscopic ultrasonography $(47 /$ $64 ; 73.44 \%)$, and minimally invasive staging (52/64; 81.25\%). Minimally invasive staging and/or positron emission tomography was performed in 57 patients $(57 / 64 ; 89.06 \%)$. The surgical approach was at the discretion of the operating surgeon. Our primary technique for esophagectomy has been previously detailed. ${ }^{20}$

\section{Tissue Handling and RNA Isolation}

Esophageal tumor tissue was obtained during surgical resection and immediately snap frozen at $-80^{\circ} \mathrm{C}$ until additional analysis. All tissues were mounted in optimal cutting temperature compound before sectioning on the cryostat. The first and last sections were mounted on slides for pathologic evaluation and intervening sections (20-30 sections; $5 \mu \mathrm{M}$ each) were cut into RNA lysis buffer (Stratagene, La Jolla, Calif). All tissues were evaluated by a pathologist to verify adequate tumor representation in the sample before additional analysis. Total RNA was extracted using the Stratagene RNA Isolation Kit according to the manufacturer's protocol. RNA quality and purity were assessed by capillary electrophoresis using an Agilent Bioanalyzer (Agilent, Santa Clara, Calif) and ultraviolet spectrophotometer (Nanodrop, Wilmington, Del).

\section{Microarray Hybridization and Raw Data Processing}

Total RNA $(1 \mu \mathrm{g})$ was labeled with biotin using a single-round in vitro transcription reaction and hybridized to Affymetrix U133 Plus 2.0 gene expression arrays in accordance with the manufacturer's recommended protocols (Affymetrix, Santa Clara, Calif). Processed microarrays were scanned using the GeneChip Scanner 7G, and the raw image files were checked visually and then processed using the Affymetrix GeneChip operating software package to obtain individual probe hybridization intensities. The guidelines for acceptable labeling and hybridization quality, as suggested by the manufacturer, were met for all arrays. Raw data were processed using the Partek Genomic Suite (Partek, St. Louis, Mo) using the gene chip robust multiarray averaging approach to provide normalized expression data for each probe set on the arrays.

\section{Gene Expression Analysis}

Microarray probe set data were filtered to exclude probe sets without a corresponding gene symbol and subsequently filtered to exclude the probe sets with low variance across the patients defined as less than the 75th percentile of probe set variance. The probe sets that passed the filter but mapped to the same gene symbol were averaged. An overall assessment of the univariate significance of the set of filtered probe sets was checked using the tail-strength statistic. The semisupervised method of Bair and Tibshirani ${ }^{21}$ was applied to the genes. Twenty thresholds, defined by the minimum absolute standardized Cox regression coefficients (Wald scores), were evaluated for significance with 10 -fold cross-validation. The threshold of 2.0 was then selected, corresponding to 59 genes. For empirical validation of the 59 genes, an unsupervised hierarchical classification was conducted, and the patients were divided into 2 groups. A set of 3 principal components was used to predict a risk score, which was used for the survival analysis. A cross-validated risk score was devised by conducting 10-fold cross-validation 100 times and averaging the patient risk scores across the 100 repetitions. We performed pathway analysis using the Ingenuity Systems and the MetaCore program.

\section{Survival Analysis}

The primary end point of the study was overall survival. Kaplan-Meier plots were constructed for estimation of overall survival, for the entire cohort and for subsets according to risk group. Risk groups were formed by stratifying the gene expression risk scores at the median to form highand low-risk categories. The log-rank test was used to analyze the differences between the 2 groups. A multivariate proportional hazards model was applied to adjust the gene signature risk classification score for pathologic T and N stage. Staging was performed according to the 6th and the 7th edition of the AJCC Cancer Staging Manual. Adequacy of the proportional hazards assumptions was checked by the correlation between the time and Schoenfeld residuals. $^{22}$ 
TABLE 1. Patient characteristics

\begin{tabular}{|c|c|}
\hline Characteristic & Value \\
\hline \multicolumn{2}{|l|}{ Gender (n) } \\
\hline Male & 54 \\
\hline Female & 10 \\
\hline \multicolumn{2}{|l|}{ Age $(y)$} \\
\hline Median & 68 \\
\hline Range & $43-88$ \\
\hline Histologic type: adenocarcinoma $(\%)$ & 100 \\
\hline \multicolumn{2}{|l|}{ Final pathologic stage (n) } \\
\hline \multicolumn{2}{|l|}{ T stage } \\
\hline $\mathrm{T} 1$ & 25 \\
\hline T1a & 5 \\
\hline $\mathrm{T} 1 \mathrm{~b}$ & 20 \\
\hline $\mathrm{T} 2$ & 8 \\
\hline $\mathrm{T} 3$ & 30 \\
\hline $\mathrm{T} 4$ & 1 \\
\hline \multicolumn{2}{|l|}{$\mathrm{N}$ stage } \\
\hline \multicolumn{2}{|l|}{ AJCC 6th edition } \\
\hline N0 & 33 \\
\hline N1 & 31 \\
\hline \multicolumn{2}{|l|}{ AJCC 7th edition } \\
\hline N0 & 33 \\
\hline N1 & 13 \\
\hline $\mathrm{N} 2$ & 9 \\
\hline $\mathrm{N} 3$ & 9 \\
\hline \multicolumn{2}{|l|}{ AJCC stage, 6th revision (n) } \\
\hline I & 20 \\
\hline IIA & 13 \\
\hline IIB & 5 \\
\hline III & 26 \\
\hline \multicolumn{2}{|l|}{ AJCC stage, 7th revision (n) } \\
\hline I & 24 \\
\hline IIA & 4 \\
\hline IIB & 8 \\
\hline IIIA & 12 \\
\hline IIIB & 15 \\
\hline IIIC & 1 \\
\hline
\end{tabular}

\section{RESULTS}

Patient Characteristics and Overall Survival Results

Esophagectomy for EAC was performed in 64 patients (54 men and 10 women), with a median age of 68 years. The histologic type was adenocarcinoma in all patients. The patient characteristics are summarized in Table 1 .

During follow-up, 30 patients were alive with a median follow-up of 34 months (range, 2-76 months). The median overall survival of the entire cohort was 27 months (lower $95 \%$ confidence interval [CI], 22 months). The estimated probability of 3-year overall survival for the entire cohort was $49 \%(95 \%$ CI, 38\%-64\%; Figure 1$)$.

\section{Gene Identification and Analysis of Gene Expression Signatures}

Gene expression was successfully assessed in the samples from all 64 patients with EAC. Adequate tumor

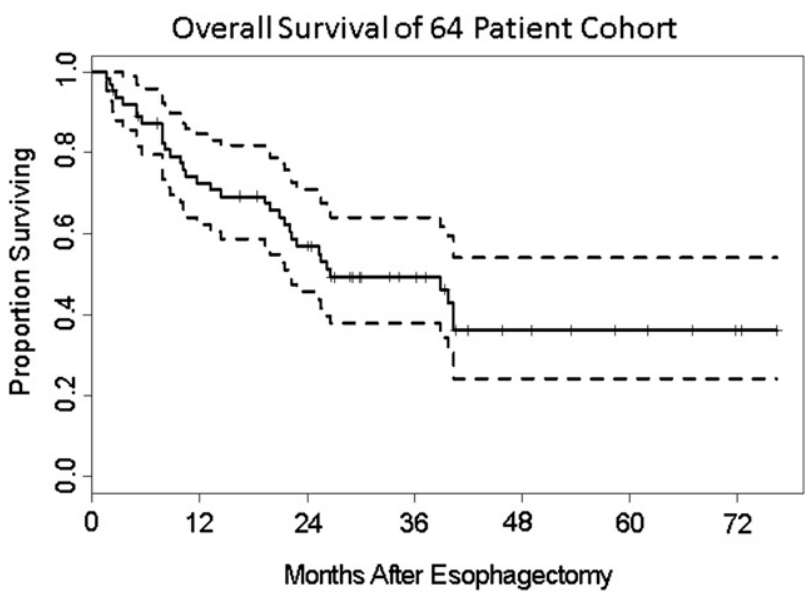

FIGURE 1. Kaplan-Meier plot of estimated overall survival for study cohort. Dotted lines denote $95 \%$ confidence band for probability of survival. Median survival was 27 months (lower 95\% confidence interval, 22 months).

representation in the specimen was ascertained before analysis, with a median tumor percentage of $80 \%$. After filtering all 54,647 Affymetrix U133 probe sets, 40,855 probe sets with an associated gene symbol were selected. These were filtered to remove the probe sets with low variance, leaving 10,214 probe sets for survival analysis. Multiple probe sets mapping to the same gene were then averaged, yielding 7425 unique gene symbols. The tail-strength statistic for these genes was 0.318 (95\% CI, 0.301-0.333), indicating a significant association of the data set as a whole with overall survival. We conducted semisupervised classification of the patients as follows. First, we calculated the principal component Wald scores for each gene. Next, we used cross-validation to step through a series of increasingly stringent thresholds to select a number of genes that appeared optimal. Choosing a threshold Wald score of 2.0 produced a list of 59 genes.

\section{Survival and Stratification by Gene Expression Signature}

Hierarchical clustering of patients and genes. We applied unsupervised hierarchical clustering with 59 genes, used to construct the risk classifier, and initially divided the 64-patient cohort into 2 clusters. That analysis (Figure 2) showed 2 well-differentiated patient clusters, identified as groups A and B. This grouping successfully stratified patients into low-risk (group A) and high-risk (group B) gene expression signatures groups for survival (Figure 3 ).

Classification and prediction of survival by gene expression signature. Using 3 principal components for the 59 genes, a risk score was calculated for each patient and divided at the median to classify the patients into 2 risk groups. The Kaplan-Meier plots of these groups (Figure 4, A) demonstrated successful classification $\left(P=7 \times 10^{-8}\right)$. Among the 32 patients identified as "lowrisk," only 7 deaths were observed. In contrast, 27 of the 32 


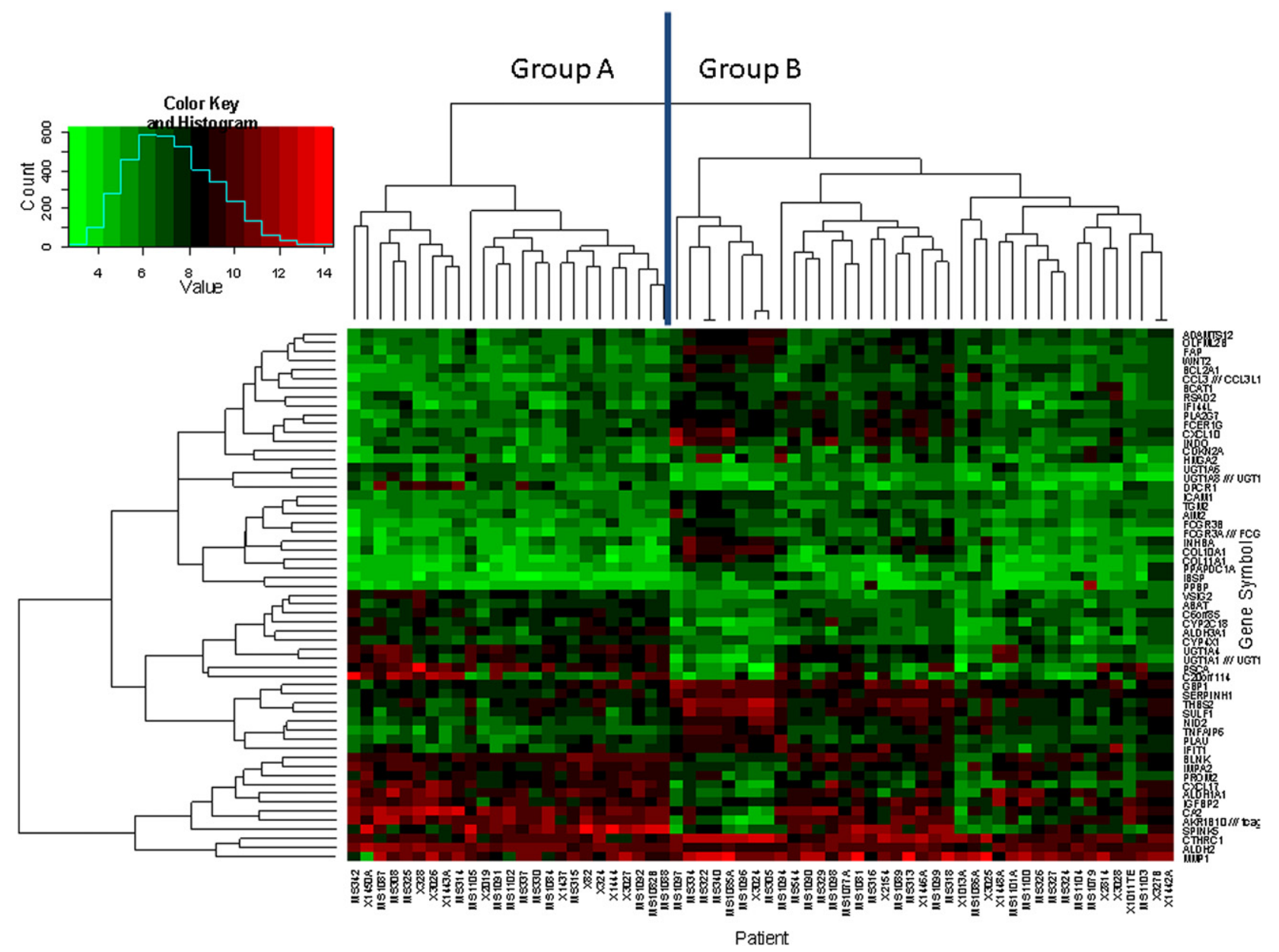

FIGURE 2. Hierarchical clustering of 59 genes selected for classification. Hierarchical clustering of gene expression data with patients in columns and genes in rows. The 59 genes selected for classification are labeled with their gene symbols. The heat map shows actual gene expression on the log 2 scale. Clustering determined by Euclidean distance of logged expression data. Column dendrogram shows patient risk groups: group A (low risk; $\mathrm{n}=25$ patients) and group $\mathrm{B}$ (high-risk; $\mathrm{n}=39$ patients).

patients in the high-risk group died. The median survival in the high-risk gene expression signature group was 19 months (95\% CI, 10-25 months) and was not reached in the lowrisk gene expression signature group. The estimated 3-year overall survival was $79 \%$ (95\% CI, 65\%-96\%) in patients with the low-risk gene expression signature and $21 \%(95 \%$ CI, $10 \%-42 \%$ ) in those with the high-risk gene expression signature. To assess the level of optimism in this estimate, we conducted a rigorous cross-validation, in which one subset of the data was used for fitting a model and a different subset of patients (who were excluded from the model construction) was used to test the model. We repeated this 10 -fold crossvalidation 100 times. The resulting log-rank test was still significant $(P=.0005$; Figure $4, B)$. The cross-validated high-risk gene expression signature group had a significantly different overall survival than the group with the low-risk signature (Figure 4, B). Of the $25 \mathrm{~T} 1$ tumors studied, $8(32 \%)$ had a high-risk gene expression signature associated with a worse prognosis. Similarly, 5 of $31(16 \%)$ node-positive patients had a low-risk gene expression signature, and 6 of $33(18 \%)$ nodenegative patients had a high-risk gene expression signature.

\section{Univariate and Multivariate Analysis of Clinical and Pathologic Factors and Gene Expression Signature}

The gene signature risk classification was tested for significant associations in a multivariate proportional hazards model that included the pathologic $\mathrm{T}$ and $\mathrm{N}$ stage. All pathologic factors, as defined in the AJCC Cancer Staging Manual, 7th edition, when considered individually, including increasing $\mathrm{T}$ and $\mathrm{N}$ stage (positive lymph nodes), were associated with increased risk. The hazard ratio (HR) for increasing T stage was 4.73 (95\% CI, 2.24-9.99) and the HR for number of positive lymph nodes (0-3 positive nodes) was 1.57 (95\% CI, 1.29-1.90). These results are summarized in Table 2. A total of 21 patients (all with nodal disease) received adjuvant chemotherapy. Owing to the selection of patients with node-positive disease, adjuvant chemotherapy was associated with a nonsignificant trend toward worse 


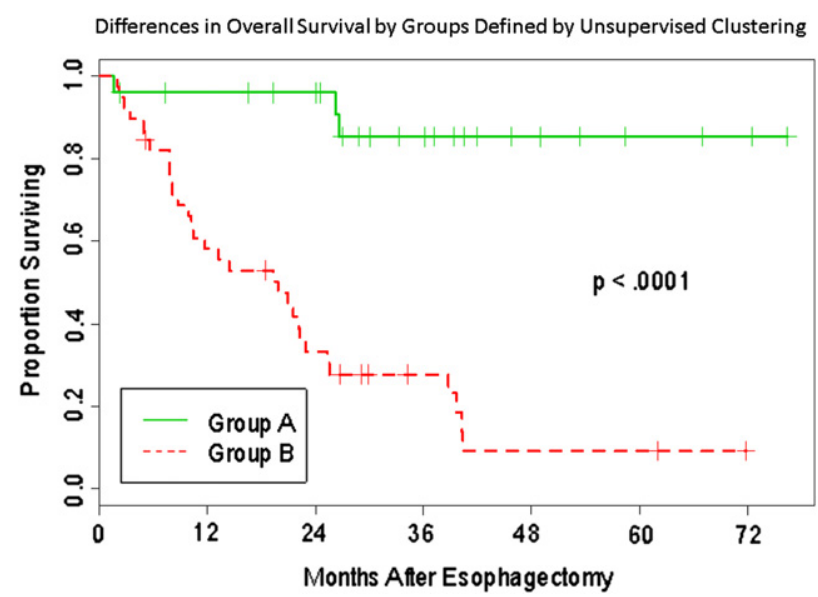

FIGURE 3. Kaplan-Meier plot of estimated overall survival stratified by gene expression profile, identified by unsupervised hierarchical clustering in Figure 2. Group A had markedly better survival than group B, corresponding to low- and high-risk gene expression profiles, respectively. The log-rank test was significant $(P<.0001)$.

survival (HR, 1.86; 95\% CI, 0.94-3.67; $P=.0753$ ). The gene expression risk category defined by the 59-gene, cross-validated risk score was strongly associated with survival, with a HR for the high- versus low-risk group of 3.46 (95\% CI, 1.64-7.29; Table 2). To assess the independence of the 59-gene signature, we adjusted the 59-gene signature risk score for the following pathologic covariates: T stage, number of positive lymph nodes (which uniquely determines the $\mathrm{N}$ stage), and tumor grade. A multivariate model of the risk group after adjusting for both $\mathrm{T}$ and $\mathrm{N}$ stage showed that the gene expression signature risk group was independently associated with survival. The adjusted HR for the high-risk gene signature group was significant, with an HR of 2.22 (95\% CI, 1.02-4.03; $P=.04$; Table 2 and Figure 5).

\section{DISCUSSION}

In the present prospective study of patients with EAC treated with esophagectomy, we identified a preliminary, 59-gene classifier that stratified patients into high-risk and low-risk prognostic categories. This internally crossvalidated prognostic signature was predictive of the outcome in patients undergoing resection for EAC. According to the HR and corresponding $P$ value on multivariate analysis, this prognostic gene signature was a strong predictor of outcome and was independent of the $\mathrm{T}$ and $\mathrm{N}$ stage.

An analysis of the pathways associated with this gene signature showed that the networks involved included those responsible for cell differentiation, cell proliferation, cellular assembly and reorganization, and those involving cell death and inflammatory responses. Inflammatory pathway genes that were significantly associated included tumor necrosis factor- $\alpha$-induced protein 6 , which is known to have an important role in the protease network, and intercellular adhesion molecule-1, which might play a role in tumor growth. ${ }^{\mathrm{A} 1-\mathrm{A} 3}$ The upregulation of inflammatory pathways has also been demonstrated previously in esophageal cancer. ${ }^{\mathrm{A} 4}$ One of the significantly associated cell cycle genes included cyclindependent kinase inhibitor 2A, which encodes p16, and acts through interaction with cyclin dependent kinase-4. ${ }^{\mathrm{A} 5, \mathrm{~A} 6}$

Differential gene expression and microRNA patterns among normal esophagus, BE, and cancer have been investigated $^{15-18,23}$; however, the prognostic value of gene expression profiles in esophageal cancer has not been frequently studied. In one study, Hao and colleagues ${ }^{18}$ evaluated the gene expression patterns in 17 patients with $\mathrm{BE}$ or EAC and compared these with those from the normal esophagus and duodenum. They found that stromal gene expression was shared between BE and EAC but not with the normal esophagus or duodenum. They emphasized the possible role of the stroma and extracellular matrix in the
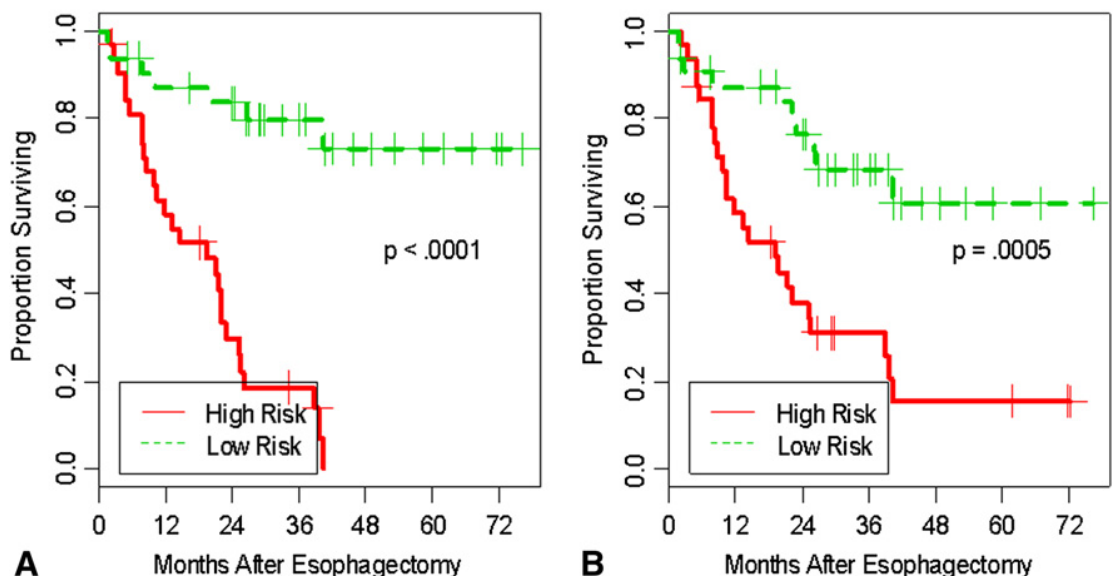

FIGURE 4. Classification of patients with supervised principle components analysis. Three principal components were used to estimate a risk score for each patient according to their expression of the 59 genes. The risk score was divided into 2 groups at the median. A, Comparison of low- and high-risk patient cohorts $(P<.0001)$. B, Same classification, using average of 100,10 -fold cross-validations to derive the risk score $(P=.0005)$. 
TABLE 2. Proportional hazards for overall survival: individual and joint covariate effects

\begin{tabular}{|c|c|c|c|c|c|c|c|}
\hline \multirow[b]{2}{*}{ Covariate } & \multirow[b]{2}{*}{ Reference } & \multicolumn{3}{|c|}{ Individual covariate effects } & \multicolumn{3}{|c|}{ Joint covariate effects } \\
\hline & & HR & $95 \% \mathrm{CI}$ & $P$ value & HR & $95 \% \mathrm{CI}$ & $P$ value \\
\hline 59-Gene signature & High vs low risk* & 3.46 & $1.64-7.29$ & .0011 & 2.22 & $1.02-4.03$ & .0438 \\
\hline $\mathrm{T}$ stage & 3-4a vs $1-2$ & 4.73 & 2.24-9.99 & $<.0001$ & 2.95 & $1.30-6.72$ & .0099 \\
\hline Positive LNs (n) & 3 vs 0 & 1.57 & $1.29-1.90$ & .0019 & 1.33 & $1.06-1.67$ & .0139 \\
\hline
\end{tabular}

$H R$, Hazard ratio; $C I$, confidence interval; $L N s$, lymph nodes. ${ }^{*}$ Defined by splitting cross-validated risk score at the median.

development of esophageal cancer. ${ }^{18}$ In another interesting study comparing BE and EAC, Dahlberg and colleagues ${ }^{16}$ evaluated gene expression in 10 samples of EAC and identified genes that were differentially expressed compared with normal controls. Selaru and colleagues ${ }^{17}$ compared 6 samples of EAC with 7 samples of BE and identified differential gene clusters in the cancer samples. Similarly, Greenawalt and coworkers ${ }^{15}$ identified differentially expressed genes when comparing BE and EAC. These studies did not, however, evaluate the outcomes associated with the differential gene expression in esophageal cancer. Tamoto and colleagues ${ }^{14}$ analyzed the gene expression profile in patients with esophageal squamous cell carcinoma and identified genes that correlated with tumor stage, although the outcomes were not specifically studied.

Gene expression profiles that classify patients into highand low-risk groups have been identified in other malignancies, such as lung, breast, and head and neck cancer. ${ }^{10-13,24}$ However, few studies have evaluated the prognostic implications of gene expression in esophageal cancer. In addition to profiling gene expression, micro-RNA expression could also add to the prediction of outcomes. ${ }^{\mathrm{A} 4}$ Hammoud

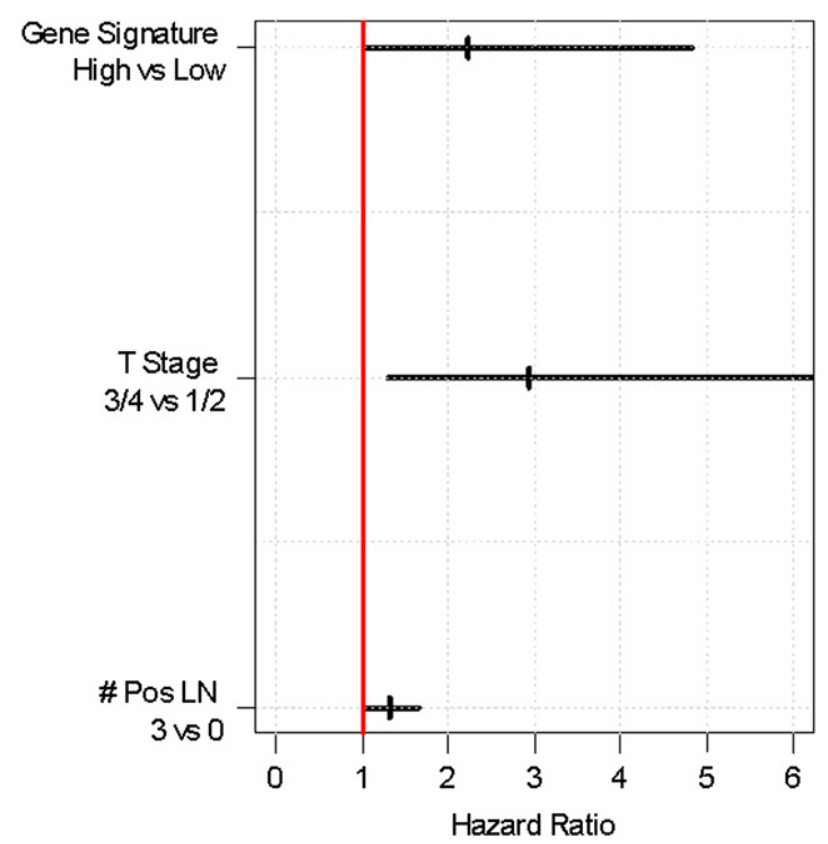

FIGURE 5. Multivariate analysis. Red vertical line depicts hazard ratio of 1 , indicating no effect. All factors remained significant at $P<.05$. Hazard ratio for gene signature, adjusting for $\mathrm{T}$ stage and $\mathrm{N}$ stage, was 2.2. and colleagues, ${ }^{19}$ in a very interesting study, evaluated gene expression in patients with EAC. They evaluated 502 genes, using a cDNA-mediated annealing, selection, extension, and ligation assay in a retrospective study of paraffinembedded tissue. With a median follow-up of 25 months, they found differential gene expression that correlated with progression and survival. Although their study was unique in many respects, its limitations included its retrospective nature, evaluation of a relatively limited number of genes compared with the DNA microarray technique, and the finding that in their data set, known predictors, such as T stage and lymph node status, did not correlate with the outcome. Despite these limitations, their study is one of the first to evaluate gene expression profiles in esophageal cancer. In another study of gene expression in EAC, 4 genes, verified by immunohistochemistry, demonstrated an association with survival. ${ }^{\mathrm{A} 7}$ Their study found that patients with all 4 of these genes dysregulated had significantly worse survival. ${ }^{\mathrm{A} 7} \mathrm{In}$ addition, integrated analysis of gene expression and comparative genomic hybridization could reveal copy number aberrations and genes with prognostic importance. ${ }^{\mathrm{A} 8}$

\section{Implications and Future Directions}

Molecular staging with evaluation of gene expression profiles in esophageal cancer has the potential to change treatment paradigms. For example, although controversial, it is commonly stated that patients with superficial tumors, in particular, intramucosal tumors, can be managed by local endoscopic excision alone. ${ }^{3}$ However, in our series, a very interesting finding was that approximately one third of the patients with superficial tumors (which included intramucosal cancer) had a high-risk gene expression profile, indicating that for these patients, the best care might be aggressive treatment.

Another interesting finding was that some patients had node-positive disease but had a favorable prognostic signature. If these results are validated further, these patients might potentially be spared aggressive, multimodality therapy. Furthermore, gene expression profiles are being investigated to predict the response to therapies. ${ }^{8,25}$ In a study evaluating the correlation between gene expression and the response to treatment (chemoradiotherapy), a prediction profile was obtained for squamous cell carcinoma but not for adenocarcinoma. ${ }^{\mathrm{A} 9}$ In another study of patients who received neoadjuvant chemoradiotherapy and resection, 2 genes demonstrated significant association with survival. ${ }^{\text {A10 }}$ In other investigations, differences were seen in expression profiling 
between patients who responded to induction chemotherapy and those who did not respond to treatment, ${ }^{25, \mathrm{~A} 11, \mathrm{~A} 12}$ suggesting that expression profiling could be of some value in distinguishing patients with differing responses to neoadjuvant therapy. In another study, angiogenesis generelated polymorphisms were related to tumor recurrence. ${ }^{\mathrm{A} 13}$

Gene expression profiles of the primary tumor can provide useful information toward our understanding of the biologic behavior of the tumor and in the selection and tailoring of therapy. The expression signatures have the potential to significantly add to traditional clinical risk factors in the prediction of outcomes. ${ }^{13}$ These findings, if validated, could lead to future clinical trials based on the gene expression profiles of the primary tumor.

\section{Strengths and Limitations}

The strengths of the present study were its prospective nature, its focus on adenocarcinoma, and the application of a standardized protocol. In addition, we rigorously internally cross-validated the gene expression signature, which was predictive of outcome, by selecting genes with a threshold Wald score of 2.0 or greater. This method allowed some control for false discovery but is not as conservative as a strict control for a fixed false discovery rate. Since we were conducting crossvalidation of candidate genes, we wanted to initially include a larger set of genes, some of which might be important but that would have been missed with a strict $P$ value criterion. Furthermore, although other studies of squamous cell cancer have been published, our study is one of the first prospective studies to evaluate the gene expression profiles in EAC. Perioperative mortality can confound the accuracy and analysis of model building using the molecular characteristics of the primary tumor. To exclude this confounding variable, we excluded all patients who died within 30 days of surgery. Cancer-specific mortality might be more specific; however, it is more difficult and less reliable to obtain during follow-up. Therefore, we used overall survival as an endpoint, similar to the endpoints used in the Worldwide Esophageal Cancer Collaboration group and the American Joint Committee on Cancer esophageal staging system. ${ }^{\mathrm{A} 14-\mathrm{A} 16}$ Moreover, the median follow-up of 34 months in our series is one of the longest reported evaluating the prognostic implications of gene expression in esophageal cancer.

Our study did have several limitations. Neoadjuvant therapy affects gene expression and confounds the analysis; therefore, we excluded patients who received neoadjuvant therapy from our study. Although this is a strength with regards to a clear gene expression analysis, a selection bias of the patients analyzed in our study might exist owing to the exclusion of the patients who received neoadjuvant therapy. The patients enrolled in the present study, however, represented a broad spectrum of patients with esophageal cancer, who underwent resection. After ascertaining that each sample had adequate tumor representation, we used the entire sample and did not use laser capture microscopy to dissect out the neoplastic cells. Although laser capture microscopy more precisely captures the neoplastic cells, a disadvantage of this method is that it ignores any stromal tissue, an important component of the tumor. ${ }^{18}$ Analyzing the entire specimen has the advantage of including the tumor, along with the stromal component, which is more representative of the in vivo tumor environment and also would be more practical in future clinical applications. It has been recognized that tumors can be multiclonal and heterogeneous, and it is possible that, despite confirming the presence of tumor on pathologic examination, all areas of heterogeneous tumors were not adequately represented.

We also acknowledge that our risk groups, as defined by the gene signature, were only strictly valid in our data set. We recognize that predictive models that are evaluated in the same data used to construct the models will be overly "optimistic" in the sense that they will seem better in the original data than in an independent set of patients. In lieu of having an independent data set, we undertook a rigorous cross-validation. In this cross-validation, we used one subset of the data for fitting a model and a different subset of patients (who were excluded from the model construction) for testing the model. This process, which was repeated (in our case 100 times), simulates the decrease in predictive ability in a new data set and is thus appropriately conservative for claiming what this signature can potentially accomplish when tested with a completely independent patient cohort. Despite the robust analysis, these results are preliminary. More work is required, and is ongoing, to refine this signature and elucidate the functional pathways that determine the survival outcomes of patients with esophageal cancer. Finally, more rigorous validation in an independent or larger set of patients is required to further refine and validate the signature.

\section{CONCLUSIONS}

In the present prospective study of gene expression in EAC, the gene expression profile was significantly associated with overall survival after esophagectomy. Furthermore, individual genes were successfully combined into a strongly predictive, internally cross-validated, gene signature. On multivariate analysis, this gene signature was independent of both tumor $(\mathrm{T})$ and nodal $(\mathrm{N})$ status. This gene expression profile is preliminary, and additional refinement and validation is needed. If validated further, these results could help stratify and select patients for appropriate treatment and direct clinical trials of neoadjuvant and adjuvant therapies for esophageal cancer.

The authors wish to thank and acknowledge the sincere efforts of the research coordinators: Ms Julie Ward, RN, Ms Peg Reamer, RN, and Ms Judy Forster, RN; Ms Cornelia Smith, BS, HT, Ms Wanda Brown, BS, and Mr Cory Riordan, BS, for the tissue handling and technical help in this project; and Ms Kathy Lovas, RHIA, for database support. The authors also thank and acknowledge Ansuman Chattopadhyay, $\mathrm{PhD}$, Health Sciences Library System at the 
University of Pittsburgh for his help and expertise with the molecular biology information service and thank Shannon Wyszomierski, PhD, University of Pittsburgh Medical Center, Pittsburgh, Pa, for editorial assistance during manuscript preparation.

\section{References}

1. Jemal A, Siegel R, Ward E, Hao Y, Xu J, Thun MJ. Cancer statistics, 2009. $C A$ Cancer J Clin. 2009;59:225-49.

2. Enzinger PC, Mayer RJ. Esophageal cancer. N Engl J Med. 2003;349:2241-52.

3. Pennathur A, Farkas A, Krasinskas AM, Ferson PF, Gooding WE, Gibson MK, et al. Esophagectomy for T1 esophageal cancer: outcomes in 100 patients and implications for endoscopic therapy. Ann Thorac Surg. 2009;87:1048-55.

4. Luketich JD, Friedman DM, Weigel TL, Meehan MA, Keenan RJ, Townsend DW, et al. Evaluation of distant metastases in esophageal cancer: 100 consecutive positron emission tomography scans. Ann Thorac Surg. 1999;68:1133-7.

5. Kaushik N, Khalid A, Brody D, Luketich J, McGrath K. Endoscopic ultrasound compared with laparoscopy for staging esophageal cancer. Ann Thorac Surg. 2007;83:2000-2.

6. May A, Günter E, Roth F, Gossner L, Stolte M, Vieth M, et al. Accuracy of staging in early oesophageal cancer using high resolution endoscopy and high resolution endosonography: a comparative, prospective, and blinded trial. Gut. 2004; 53:634-40.

7. Quackenbush J. Microarray analysis and tumor classification. $N$ Engl J Med. 2006;354:2463-72

8. Shimada Y, Sato F, Shimizu K, Tsujimoto G, Tsukada K. cDNA microarray analysis of esophageal cancer: discoveries and prospects. Gen Thorac Cardiovasc Surg. 2009;57:347-56.

9. Gupta VK, Feber A, Xi L, Pennathur A, Wu M, Luketich JD, Godfrey TE. Association between CCND1G/A870 polymorphism, allele-specific amplification, cyclin D1 expression, and survival in esophageal and lung carcinoma. Clin Cancer Res. 2008;14:7804-12.

10. Beer DG, Kardia SL, Huang CC, Giordano TJ, Levin AM, Misek DE, et al. Geneexpression profiles predict survival of patients with lung adenocarcinoma. Nat Med. 2002;8:816-24.

11. Roepman P, Lodewyk F, Wessels A, Kettelarij N, Kemmeren P, Miles AJ, et al. An expression profile for diagnosis of lymph node metastases from primary head and neck squamous cell carcinomas. Nat Genet. 2005;37:182-6.

12. van't Veer LJ, Dai H, van de Vijver MJ, He YD, Hart AA, Mao M, et al. Gene expression profiling predicts clinical outcome of breast cancer. Nature. 2002; 415:530-6.

13. Huang E, Cheng SK, Dressman H. Gene Expression predictors of breast cancer outcomes. Lancet. 2003;361:1590-6.

14. Tamoto E, Tada M, Murakawa K, Takada M, Shindo G, Teramoto K, et al. Geneexpression profile changes correlated with tumor progression and lymph node metastasis in esophageal cancer. Clin Cancer Res. 2004;10:3629-38.

15. Greenawalt DM, Duong C, Smyth GK, Ciaverella ML, Thompson NJ, Tiang T, et al. Gene expression profiling of esophageal cancer: comparative analysis of Barrett's esophagus, adenocarcinoma, and squamous cell carcinoma. Int J Cancer. 2007;120:1914-21.

16. Dahlberg PS, Ferrin LF, Grindle SM, Nelson CM, Hoang CD, Jacobson B. Gene expression profiles in esophageal adenocarcinoma. Ann Thorac Surg. 2004;77:1008-15.

17. Selaru FM, Zou T, Xu Y, Shustova V, Yin J, Mori Y, et al. Global gene expression profiling in Barrett's esophagus and esophageal cancer: a comparative analysis using cDNA microarrays. Oncogene. 2002;21:475-8.

18. Hao Y, Triadafilopoulos G, Sahbaie P, Yound HS, Omary MB, Lowe AW. Gene expression profiling reveals stromal genes expressed in common between Barrett's esophagus and adenocarcinoma. Gastroenterology. 2006;131:925-33.

19. Hammoud ZT, Badve S, Zhao Q, Li L, Saxena R, Thorat MA, et al. Differential gene expression profiling of esophageal adenocarcinoma. J Thorac Cardiovasc Surg. 2009; 137:829-34.

20. Luketich JD, Alvelo-Rivera M, Buenaventura PO, Christie NA, McCaughan JS, Litle VR, et al. Minimally invasive esophagectomy: outcomes in 222 patients. Ann Surg. 2003;238:486-95.

21. Bair E, Tibshirani R. Semi-supervised methods to predict patient survival from gene-expression data. PLOS Biol. 2004;2:511-22.

22. Grambsch P, Therneau T. Proportional hazards tests and diagnostics based on weighted residuals. Biometrika. 1994;81:515-26.

23. Feber A, Xi L, Luketich JD, Pennathur A, Landreneau RJ, Wu M, et al. MicroRNA expression profiles of esophageal cancer. J Thorac Cardiovasc Surg. 2008; 135:255-60.
24. Houng CD, D'Cunha J, Tawfic SH, Gruessner AC, Kratzke RA, Maddaus MA. Expression profiling of non-small cell lung carcinoma identifies metastatic genotypes based on lymph node tumor burden. J Thorac Cardiovasc Surg. 2004;127:1332-41.

25. Luthra R, Wu T-T, Luthra MG, Izzo J, Lopez-Alvarez E, Zhang L, et al. Gene expression profiling of localized esophageal carcinomas: association with pathologic response to preoperative chemoradiation. J Clin Oncol. 2006;24:259-67.

\section{Appendix: Additional References}

A1. Gho YS, Kim PN, Li HC, Elkin M, Kleinman HK. Stimulation of tumor growth by human soluble intercellular adhesion molecule-1. Cancer Res. 2001;61:4253-7.

A2. Roland CL, Harken AH, Sarr MG, Barnett CC Jr. ICAM-1 expression determines malignant potential of cancer. Surgery. 2007;141:705-7.

A3. Available from: http://www.ncbi.nlm.nih.gov/gene/7130\#reference-sequences.

A4. Nguyen GH, Schetter AJ, Chou DB, Bowman ED, Zhao R, Hawkes JE, et al. Inflammatory and microRNA gene expression as prognostic classifier of Barrett's-associated esophageal adenocarcinoma. Clin Cancer Res. 2010;16: 5824-34.

A5. Hu N, Wang C, Su H, Li WJ, Emmertt-Buck MR, Li G, et al. High frequency of CDKN2A alterations in esophageal squamous cell carcinoma from a high-risk Chinese population. Genes Chromosomes Cancer. 2004;39:205-16.

A6. National Center for Biotechnology Information. Available from: http://www. ncbi.nlm.nih.gov/gene/1029\#reference-sequences. Accessed .

A7. Peters CJ, Rees JR, Hardwick RH, Hardwick JS, Vowler SL, Ong CA, et al. A 4-gene signature predicts survival of patients with resected adenocarcinoma of the esophagus, junction, and gastric cardia. Gastroenterology. 2010;139: 1995-2004.

A8. Goh XY, Rees JR, Paterson AL, Chin SF, Marioni JC, Save V, et al. Integrative analysis of array-comparative genomic hybridisation and matched gene expression profiling data reveals novel genes with prognostic significance in oesophageal adenocarcinoma. Gut. 2011;60:1317-26.

A9. Duong C, Greenawalt DM, Kowalczyk A, Ciavarella ML, Raskutti G, Murray WK, et al. Pretreatment gene expression profiles can be used to predict response to neoadjuvant chemoradiotherapy in esophageal cancer. Ann Surg Oncol. 2007;14:3602-9.

A10. Kim SM, Park Y-Y, Park ES, Cho JY, Izzo JG, Zhang D, et al. Prognostic biomarkers for esophageal adenocarcinoma identified by analysis of tumor transcriptome. PLoS One. 2010;5:e15074.

A11. Schauer M, Janssen KP, Rimkus C, Raggi M, Feith M, Friess H, Theisen J. Microarray-based response prediction in esophageal adenocarcinoma. Clin Cancer Res. 2010;16:330-7.

A12. Maher SG, Gillham CM, Duggan SP, Smyth PC, Miller N, Muldoon C, et al. Gene expression analysis of diagnostic biopsies predicts pathological response to neoadjuvant chemoradiotherapy of esophageal cancer. Ann Surg. 2009;250:729-37.

A13. Lurje G, Leers JM, Pohl A, Oezcelik A, Zhang W, Ayazi S, et al. Genetic variations in angiogenesis pathway genes predict tumor recurrence in localized adenocarcinoma of the esophagus. Ann Surg. 2010;251:857-64.

A14. Rice TW, Rusch VW, Apperson-Hansen C, Allen MS, Chen LQ, Hunter JG, et al. Worldwide Esophageal Cancer Collaboration. Dis Esophagus. 2009;22:1-8.

A15. Edge SB, Byrd DR, Compton CC, Fritz AG, Greene FL, Trotti A, eds. AJCC cancer staging manual. 7th ed. New York: Springer-Verlag; 2009:103-15.

A16. Rice TW, Blackstone EH, Rusch VW. A cancer staging primer: esophagus and esophagogastric junction. J Thorac Cardiovasc Surg. 2010;139:527-9.

\section{Discussion}

Dr Chuong D. Hoang (Stanford, Calif). I have nothing to disclose.

Dr Pennathur and his group present a timely study showing that traditional diagnostic and prognostic factors erroneously stratify some patients some of the time, and I think his group has taken a step forward into the future direction of personalized cancer care. To be relevant, however, molecular prediction has to provide additional information, independent of traditional criteria or provide prognostic information within the subgroups defined by those traditional criteria. In this context, I have the following 3 questions.

First, could you please clarify whether any patients in your study received neoadjuvant chemoradiotherapy, and, if so, how 
were you able to retrieve in all 69 resection specimens more than $70 \%$ tumor representation for subsequent array profiling? Is it not surprising, here, that there were no pathologic complete responders in your prospective study?

Dr Pennathur. Thank you, Dr Hoang, for your kind comments.

The first question, patients who had neoadjuvant chemotherapy or radiotherapy were excluded from the study because those have an effect on gene expression. These patients are patients who were not treated with neoadjuvant therapy.

Dr Hoang. Including the T3, T4 that you mentioned?

Dr Pennathur. Yes, including the patients who had T3 in the final pathology analysis.

Dr Hoang. Question 2, the 2-dimensional heat map of your gene classifier showed that there was imperfect representation of some of the high-risk patients who were grouped into the lowrisk gene signature and vice versa. What was the specific error rate in your prognostication? How did your gene classifier compare with the old and now the new TNM staging systems for esophageal cancer? Furthermore, were you able to segregate by stage how your classifier performed?

Dr Pennathur. Again, thank you for those questions.

The purpose of the heat map classifier was to investigate whether any gene pattern exists with the potential to classify patient risk, and the division into 2 groups (A and B) was based purely on the empirical clustering of the patients. The specific error rate of classification cannot be found in this context because we used survival as the endpoint—not class-and illustrated the classification accuracy, not with an error rate but with survival differences between groups.

The gene risk signature was independent of the pathologic stage of the prior system. Regarding the questions about the new staging system, we have not yet actually compared this gene signature with the new staging system. We do plan on performing an analysis with the new staging system and will include this in the manuscript.

Dr Hoang. My final and third question is, by choosing a microarray-based prediction method, how do you plan to overcome some of the inherent weaknesses in this technology that have limited its widespread application in daily clinical tests? This includes that microarray tests are labor-intensive, time-consuming, and expensive, there are issues with reproducibility, and, usually, these tests include many numbers of genes, making data handling impractical and cumbersome most of the time.

Dr Pennathur. I think that is a very relevant question. I think these technologies are constantly evolving. As it stands, it is labor intensive, and of course, the cost is a factor. We believe that over time, as this technology evolves, the cost factor could be addressed, and anticipate that it will decrease. Also, ultimately I think it is going to be a combination of molecular staging, clinical staging, and known prognostic factors, which is going to help determine the prognosis of the patient. I think if it really allows us to choose a personalized treatment for a particular patient in terms of the resection we are doing, in terms of the selection for multimodality treatment for the individual patient that translates to improved outcomes, then I think the efforts and costs are worth it, but I agree that currently the costs are quite high, and as the technology improves, we think the cost will come down.

Dr Hoang. Thank you.

Dr Sandro Mattioli (Bologna, Italy). If I understood you, you have demonstrated that your gene patterns do correspond to higher stage tumors, which is good, but you are surely aware that some tumors grow slowly and this would not help your aims. But the major criticism is that you have put together apples and oranges, because you did not define the esophageal adenocarcinoma. Not all esophageal adenocarcinomas are the same. Some stem from one origin; some others stem from another origin. You did not show how many of those cancers were associated with BE or not. Thus, I think you are very far from your aiming point.

Dr Pennathur. Thank you for your comments. With regard to your second question, we presume that most of these cancers came from $\mathrm{BE}$; however, this was not done as a separate analysis. Our aim in the current study was to investigate the gene expression profiles in all patients with esophageal adenocarcinoma who underwent esophagectomy with criteria as detailed in our inclusion/exclusion criteria, and not restrict this to specific stages or its specific origin; so we are on target with the aims of the current study. We appreciate your comments and will consider your suggestions for a future study. With regard to your initial question and statement - regarding the pathologic stage and gene expression-the gene classifier predicts risk in all stages, for example, predicts high-risk T1 patients as well as lowrisk T3 patients. For example, even in the high-risk group, there were a lot of superficial tumors- $-\mathrm{T} 1$ patients and $\mathrm{T} 2$ patients, approximately $50 \%$ of them in the high-risk group were $\mathrm{T} 1$ and $\mathrm{T} 2$ patients, and roughly one third of T1 patients were in the high-risk group. So, the high-risk gene signature was not exclusively seen in the higher stage tumors. In fact, the gene risk signature was independent of the pathologic stage in these patients. So, I think there is a gene signature encoded early on that determines the survival in these patients.

Dr Keshavjee. I think that was the most important part of your presentation, that high-risk group did represent some $\mathrm{T} 1$ and $\mathrm{T} 2$ and N0 patients, and that is unique.

Dr Thomas M. Egan (Chapel Hill, NC). You showed elevated expression and reduced expression, but compared to what? Did you have a control?

Dr Pennathur. That is a great question. We reviewed some of the DNA microarray literature; our gene arrays had an implicit control in the sense that arrays are normalized to the average array. Differential expressions between groups are then relative but because all arrays are normalized, comparisons are meaningful. Recognizable gene differences are predicated on both fold changes (ratios of differences) and low $P$ values.

Dr Egan. But how can you identify those a priori? Was it based on their survival?

Dr Pennathur. We use our model to predict each patient's risk of death and then use the median of estimated risk to divide them into high-risk and low-risk groups.

Dr Egan. The other question is related to the potential for sampling error. How did you know you were just getting tumor versus tumor and underlying tissue?

Dr Pennathur. I think that is a great question. We made sure that the pathologists, who were co-investigators, examined the tumor to ensure there was more than $70 \%$ tumor representation, and we believe the tumor tissue, along with the stromal tissue, has an important bearing on how the tumor behaves. That is why we did not use, for example, laser capture microscopy and we took the gross tumor, which we believe is more representative.

Thank you for your comments. We thank the Association for the opportunity to present this paper. 